\title{
Sol-gel derived CdS nanocrystalline thin films: optical and photoconduction properties
}

\author{
Ziaul RaZa Khan ${ }^{1, *}$, Munirah $^{2}$, Anver AzIZ $^{2}$, Mohd. Shahid Khan ${ }^{2}$ \\ ${ }^{1}$ Department of Physics, College of Science, University of Ha'il, P.O. Box-2440, Ha'il, Saudi Arabia \\ ${ }^{2}$ Laser Spectroscopy Lab, Department of Physics, Jamia Millia Islamia, New Delhi-110025, India
}

\begin{abstract}
High-quality CdS nanocrystalline thin films were grown by sol-gel spin coating method at different solution temperatures on glass substrates. As-deposited films exhibited nanocrystalline phase with hexagonal wurtzite structure and showed good adhesion and smooth surface morphology. It was clearly observed that the crystallinity of the thin films improved with the increase in solution temperature. Crystallites sizes of the films also increased and were found to be in the range of $10 \mathrm{~mm}$ to $17 \mathrm{~nm}$. The influence of the growth mechanism on the band and sub-band gap absorption of the films was investigated using UV-Vis and photothermal deflection spectroscopy (PDS). The band gap values were calculated in the range of $2.52 \mathrm{eV}$ to $2.75 \mathrm{eV}$. The band gap decreased up to $9 \%$ with the increase in solution temperature from $45{ }^{\circ} \mathrm{C}$ to $75{ }^{\circ} \mathrm{C}$. Absorption coefficients estimated by PDS signal showed the significant absorption in low photon energy region of $1.5 \mathrm{eV}$ to $2.0 \mathrm{eV}$. The dark and illuminated I-V characteristics revealed that the films were highly photosensitive. The results demonstrated the potential applications of sol-gel grown CdS nanocrystalline thin films as photoconductors and optical switches.
\end{abstract}

Keywords: CdS; sol-gel; optical properties; photothermal deflection spectroscopy; photoconductivity

\section{Introduction}

Recently, an increasing interest has been devoted to the development of II-VI group binary inorganic low dimensional materials via simple and inexpensive chemical route. This kind of material is highly desired for various potential applications in the field of advanced electronic and optoelectronic devices. CdS is one of important candidates of II-VI group binary inorganic semiconductors for optoelectronic applications. It has direct band gap of $2.42 \mathrm{eV}$ at room temperature and high photosensitive properties [1-5]. CdS nanocrystalline thin films have recently received much attention because of their unique physical, chemical and mechanical properties as compared to the bulk counterpart [6-8]. They are extensively used in optoelectronic devices because they tune emission in the visible region of the electromagnetic spectrum by the change in particle size. CdS thin films are also useful in the fabrication of thin film transistors, light detectors, solar cells [9-11]. Fundamental

*E-mail: zrazakhan05@gmail.com properties of a semiconducting material, such as optical, electrical and magnetic can be tailored by controlling the size, structure and surface states of nanocrystalline materials.

Optical absorption in low-dimensional semiconducting materials has attracted much attention due to their significant applications especially in light detecting and trapping devices [12]. Therefore, the exact value of absorption coefficient of semiconductor nanocrystalline thin films plays a major role in the fabrication of efficient devices. Recently, photothermal deflection spectroscopy has emerged as a powerful tool for the determination of optical absorption of nanomaterials with a high precession. It is versatile technique and can be successfully applied in the measurement of absorption coefficients of semiconductor thin films or the investigation of density of gap states in inorganic and organic semiconductor thin films. The PDS technique is mainly based on the heating of the samples under investigation by a normally incident beam of monochromatic light, inducing a gradient of refractive index in the surroundings of the medium. Light 
from a probe laser, essentially grazing the surface of the material, experiences a periodic deflection synchronous with the modulation in the intensity of the light from the pump. The amplitude and phase of this periodic deflection can be monitored with a position sensor and a differential AC synchronous detection scheme. If the wavelength of the pump light is varied, the deflection of the probe laser becomes a measure of an optical absorption spectrum of the material. The relation between amplitude of PDS signal A and absorption coefficient $\alpha$ is given by $[13,14]$ :

$$
A=A_{\text {sat }}(1-\exp (-\alpha l))
$$

where A is the PDS signal amplitude, 1 is the thickness of the film and $A_{\text {sat }}$ is the saturated amplitude which depends on the geometrical parameters of the experimental setup. On the other hand, $\mathrm{CdS}$ also has photoresistive properties. The resistance of photoresistor changes with the light intensity. With the increase in light intensity, resistance of the film decreases. It exhibits the photoconductive properties.

In the present work, high quality nanocrystalline $\mathrm{CdS}$ thin films were grown on glass substrates by sol-gel spin coating method at different solution temperatures. Structural and morphological properties of thin films were studied from XRD spectra and FE-SEM micrographs. The influence of solution temperature on the band gap and sub-band gap was investigated by UV-Vis and PDS. Photoconductive properties of the films were investigated in details.

\section{Experimental}

\subsection{Growth and characterization of sam- ples}

CdS nanocrystalline thin films were deposited on glass substrates by sol-gel spin coating method. The size of substrate was $1.5 \mathrm{~cm}^{2} \times 1.5 \mathrm{~cm}^{2}$. All chemicals, purchased from Merc Co., were of analytical grade with the purity of $99.99 \%$. First, we have prepared $\mathrm{CdS}$ sols for spin coating by dissolving cadmium acetate $\left(\mathrm{CH}_{3} \mathrm{COO}\right)_{2} \mathrm{Cd} \cdot 2 \mathrm{H}_{2} \mathrm{O}$ and thiourea $\left(\mathrm{NH}_{2} \mathrm{CSNH}_{2}\right)$ as $\mathrm{Cd}$ and $\mathrm{S}$ sources, respectively, in 2-methoxyethanol separately and kept them under constant stirring for $1 \mathrm{~h}$. Then, we mixed both the solutions and stirred for half an hour at $45^{\circ} \mathrm{C}, 55^{\circ} \mathrm{C}, 65^{\circ} \mathrm{C}$ and $75^{\circ} \mathrm{C}$, separately. The cadmium acetate and thiourea concentration were $1.0 \mathrm{M}$. The molar ratio of $\mathrm{Cd} / \mathrm{S}$ was kept constant at unity. Monoethanolamine (MEA: $\mathrm{H}_{2} \mathrm{~N}\left(\mathrm{CH}_{2}\right)_{2} \mathrm{OH}$ ) was added to obtain the desired sols. Solution was aged for $3 \mathrm{~h}$ to gain the thermal stability. Secondly, the glass substrates were cleaned using detergent and washed ultrasonically with acetone and distilled water. The glass substrates were kept in an oven at $85{ }^{\circ} \mathrm{C}$ for $15 \mathrm{~min}$. Fresh solution was used for fabrication of thin films at $3000 \mathrm{rpm}$ for $30 \mathrm{~s}$. This process was repeated for seven times to increase the film thickness. Finally, to remove the defects and organic unwanted particles, the $\mathrm{CdS}$ thin films were post-annealed at $200{ }^{\circ} \mathrm{C}$ for $2 \mathrm{~h}$ in the $\mathrm{N}_{2}$ atmosphere. The grown nanocrystalline thin films are shown in Fig. 1. The chemical reactions which took place in preparing the parental solution were as follows [15]:

$$
\mathrm{Cd}\left(\mathrm{CH}_{3} \mathrm{COO}\right)_{2} \rightarrow \mathrm{Cd}^{2+}+2(\mathrm{CHCOO})^{-}
$$

$$
\begin{gathered}
\mathrm{Cd}^{2+}+n \mathrm{HO}(\mathrm{CH})_{2} \mathrm{NH}_{2} \rightarrow \mathrm{Cd}\left[\mathrm{O}\left(\mathrm{CH}_{2}\right)_{2} \mathrm{NH}_{2}\right]^{2-n} \\
\mathrm{NH}_{2} \mathrm{CSH}_{2} \mathrm{~N} \rightarrow\left(\mathrm{NH}_{2}\right)_{2} C^{2+}+\mathrm{S}^{2-} \\
\quad \mathrm{Cd}\left[\mathrm{O}\left(\mathrm{CH}_{2}\right)_{2} \mathrm{NH}_{2}\right]^{2-n}+\mathrm{S}^{2-} \\
\rightarrow \mathrm{Cd} S+n \mathrm{HO}\left(\mathrm{CH}_{2}\right)_{2} \mathrm{NH}_{2}
\end{gathered}
$$

In the reactions, solvent acts as a catalyst to carry out the reactions. Firstly, mediate cadmium complex is formed. The sulphide ions interact with the cadmium complex to form CdS. The controlled growth of $\mathrm{CdS}$ was achieved using the chemical precursors comparatively to the other inorganic chemical precursors. After post-annealing the unwanted organic parts were evaporated. The ready samples were characterized using different characterization techniques. The structural properties of the films were investigated using Panalytical X-ray diffractometer model PW3710. Optical absorbance spectra of the films were recorded 
with a double beam spectrophotometer model JASCOV570 in the range of $250 \mathrm{~nm}$ to $1100 \mathrm{~nm}$. Surface morphology was examined by field emission electron microscope model Carl-ZEISS EVO40. For measurement of absorption coefficient, tungsten-halogen lamp was used to pump the sample, which generated heat within and around the sample surface. The monochromator was used to prevent light dispersion. Light beam, after passing by the monochromator was further passed through the low-pass filter and then focused on the sample. The light was mechanically chopped at $20 \mathrm{~Hz}$. The $\mathrm{CCl}_{4}$ liquid was used as a surrounding medium, due to its very high coefficient of variation of refractive index with temperature $(\delta \mathrm{n} / \delta \mathrm{T}=$ $\left.5 \times 10^{-4} \mathrm{~K}^{-1}\right)$. To confirm that the surrounding material does not influence the film surface and signals, the optical absorbance of films immersed in $\mathrm{CCl}_{4}$ for $5 \mathrm{~h}$ was also measured which did not show any significant change. The He-Ne laser with the power of $5 \mathrm{~mW}$ was used as a probe beam, just to graze the sample surface. The lock-in amplifier was used to amplify the detector signal. The signal was recorded using four-quadrant detector. First, silver electrodes were deposited on the films by thermal evaporator at a vacuum of $1.22 \times 10^{-3} \mathrm{~Pa}$. The gap between two electrodes was $2 \mathrm{~mm}$ for measurement of photoconduction properties. The dark and illuminated I-V measurements were made using DC Keithley 487 multimeter. White light lamp of $200 \mathrm{~W}$ was used for illumination of the samples, and to avoid heating effect in the samples, the lamp was placed at a height of $35 \mathrm{~cm}$.

\section{Results and discussion}

\subsection{X-ray diffraction analysis}

XRD spectra of CdS nanocrystalline thin films obtained by using sol-gel spin coating method at different solution temperatures are shown in Fig. 2. It has clearly been observed that the crystallites and crystallinity of CdS thin films has changed with the change in solution temperature. The films grown at $45^{\circ} \mathrm{C}$ and $55^{\circ} \mathrm{C}$ show a broad hump at an angle of $26.38^{\circ}$. No sharp peak is observed. The films grown at $65{ }^{\circ} \mathrm{C}$ and $75^{\circ} \mathrm{C}$ confirm crystalline nature with preferred orientation along ( 002 2) plane at an angle $26.38^{\circ}$. It is in a good agreement with the hexagonal structure of CdS thin films [16]. The sample grown at $75{ }^{\circ} \mathrm{C}$ shows superior crystalline nature in comparison to the films grown at lower temperatures. The crystallite size of the films was calculated using Debye-Scherer formula and the value of crystallites size was found to be in the range of $10 \mathrm{~nm}$ to $17 \mathrm{~nm}$. In addition, the XRD spectra reveal that the films are nanocrystalline in nature and the crystallite size of the films is increasing with the increasing in solution temperature. The increase in crystallites size may be due to the enhancement in the chemical reaction rate and rapid nucleation of $\mathrm{CdS}$ nanocrystals with the increase in temperature.

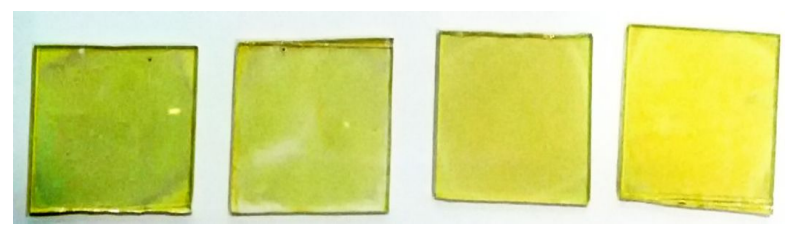

Fig. 1. Sol-gel grown CdS nanocrystalline thin films at different solution temperatures.

\subsection{FE-SEM analysis}

Micrographs of CdS nanocrystalline thin films are shown in Fig. 3. From Fig. 3 it is observed that the films have smooth, compact morphology and are free from voids and cracks. The micrographs also show good adhesion to the substrates. The morphology of the films changes significantly with changing solution temperature. The films grown at $45{ }^{\circ} \mathrm{C}$ do not show clear round shapes but tightly packed nanocrystals with the size of few nanometres. The films grown at $75^{\circ} \mathrm{C}$ solution temperature have similar shape and morphology of particles but the size of the particles increased, which can be observed clearly. It is also confirmed by XRD results analysis.

\subsection{UV-Vis analysis}

Room temperature absorbance spectra of CdS nanocrystalline thin films in the range of $250 \mathrm{~nm}$ to $1100 \mathrm{~nm}$ are shown in Fig. 4. From Fig. 4, the influence of solution temperature on absorbance spectra 


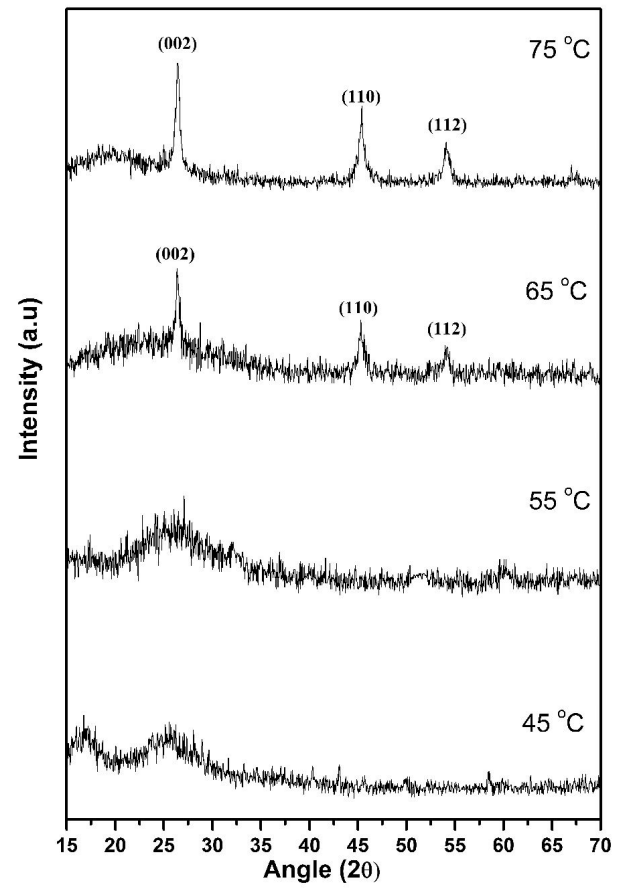

Fig. 2. XRD patterns of CdS nanocrystalline thin films at different solution temperatures.
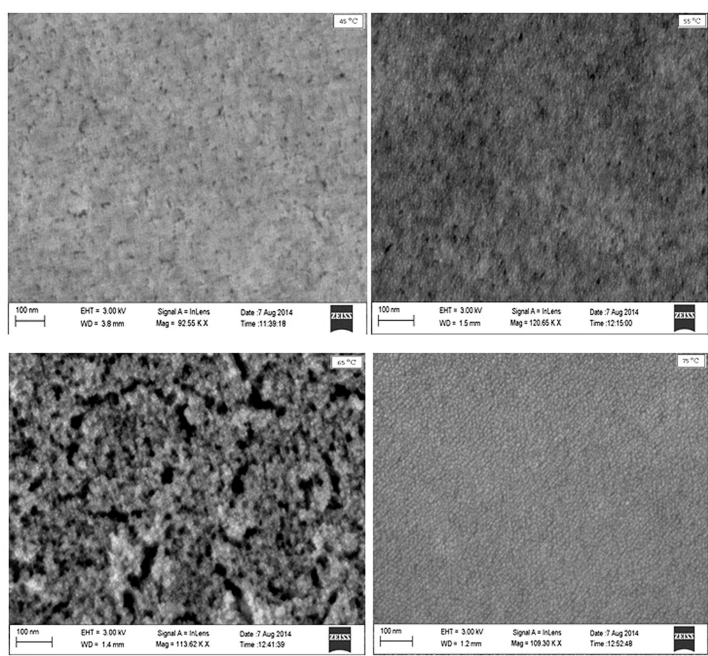

Fig. 3. FE-SEM micrographs of CdS nanocrystalline thin films obtained at different solution temperatures.

is clearly observed. All the samples exhibit a sharp band edge at $450 \mathrm{~nm}$ to $500 \mathrm{~nm}$; this confirms the formation of uniform nanoparticles. Absorption coefficients $\alpha$ were calculated using equation 6 [17]:

$$
\alpha=\frac{O D}{d}
$$

where OD is the optical density and $\mathrm{d}$ is the film thickness. The high absorption region of $2.5 \mathrm{eV}$ to $3.0 \mathrm{eV}$ is governed by the Urbach relation:

$$
(\alpha h v)=A\left(h v-E_{g}\right)^{p}
$$

where $\mathrm{A}$ is a function of index of refraction and hole/electron effective masses. The band gap of thin films was estimated from the Tauc plots. The Tauc plots of thin films $(\alpha \mathrm{h} v)^{2}$ vs. hv are shown in Fig. 5. Direct optical band gap was determined by extrapolating the straight line to the zero absorption coefficient on photon energy axis of $(\alpha \mathrm{h} v)^{2}$ vs. hv graph. The value of band gap of the films was found to be $2.57 \mathrm{eV}$ to $2.75 \mathrm{eV}$ which is well agreed with the earlier reported values [18-20]. All the thin films have higher band gap values than the bulk CdS material. It may be due to the quantum confinement effect occurring in the CdS thin films. In addition, an influence of solution temperature on band edge was observed. On increasing the solution temperature, the band gap of the films decreases. It confirms that the increase in particle size with an increase in solution temperature, hence, the band gap decreases. The particles size was also determined using the Brus equation [15]:

$$
\Delta E_{g}=\frac{h^{2} \pi^{2}}{2 R^{2}}\left(\frac{1}{m_{e}^{*}}+\frac{1}{m_{h}^{*}}\right)-\frac{1.8 e^{2}}{4 \pi \varepsilon \varepsilon_{0} R}
$$

where $\mathrm{m}_{\mathrm{e}}^{*}$ and $\mathrm{m}_{\mathrm{h}}^{*}$ are the effective masses of an electron in the conduction band and a hole in the valence band, respectively, $h$ is the Planck constant, $e$ is the electron charge, $\epsilon_{0}$ is the permittivity of free space $\left(8.854 \times 10^{-12} \mathrm{C}^{2} \cdot \mathrm{N}^{-1} \cdot \mathrm{m}^{-2}\right), \epsilon$ is the relative permeability (5.7), $\mathrm{E}_{\mathrm{g}}$ is the energy gap of bulk CdS, and R is the particle size. Particle size estimated by Brus equation 8 shows a good agreement with the particle size determined from FE-SEM results.

\subsection{PDS analysis}

The absorption coefficients of CdS nanocrystalline thin films deduced from PDS measurements are shown in Fig. 6. The values of absorption coefficients $\alpha$ were calculated from PDS signal using equation 6. From Fig. 6, it can be observed 


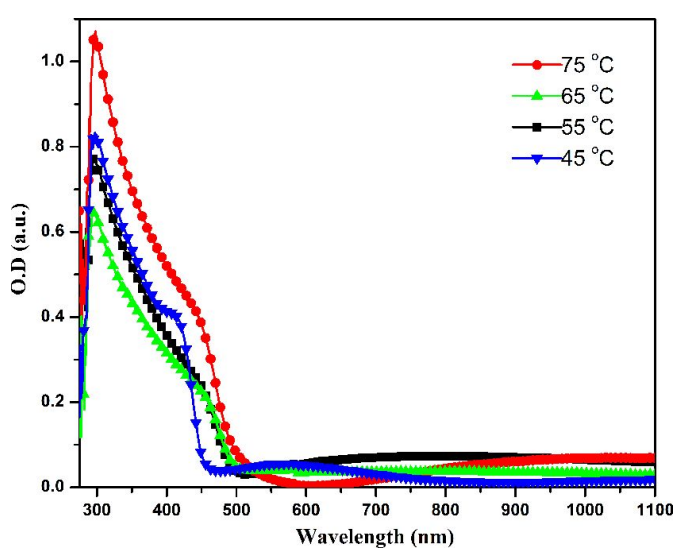

Fig. 4. Absorbance spectra of CdS nanocrystalline thin films obtained at different solution temperatures.

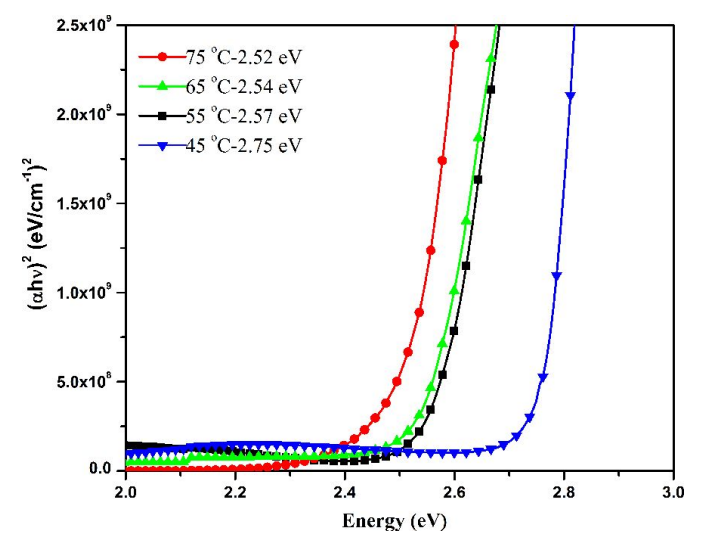

Fig. 5. Plots of $(\alpha \mathrm{h} v)^{2}$ vs. hv of CdS nanocrystalline thin films obtained at different solution temperatures.

that two saturation regions exist in high and low photon energy regions. In the band gap region, absorption coefficients become saturated due to high value of absorption coefficients. In forbidden energy gap region, the films reveal weak absorption; it may be attributed to the defects, grain boundaries and extended states of nanocrystals of the thin films. Two main factors suggest a weak absorption: one is dangling bond like defects and the second major reason is the accumulation of impurities and adsorbate at the surface of thin films during the growth process $[21,22]$. In high energy region of $2.4 \mathrm{eV}$ to $3.0 \mathrm{eV}$, absorption coefficients of thin films have the same value like in traditional UV-Vis spectroscopy. The influence of solution temperature on sub-band gap (weak absorption) of the films was also investigated. Overall absorption in the sub-band gap region was high for the thin film grown at $75{ }^{\circ} \mathrm{C}$. It may be due to the enhancement in surface defects or extended states of the grown nanocrystals with the increase in solution temperature. Weak absorption in forbidden energy region can play an important role in designing optoelectronic devices based on sol-gel grown $\mathrm{CdS}$ nanocrystalline thin films.

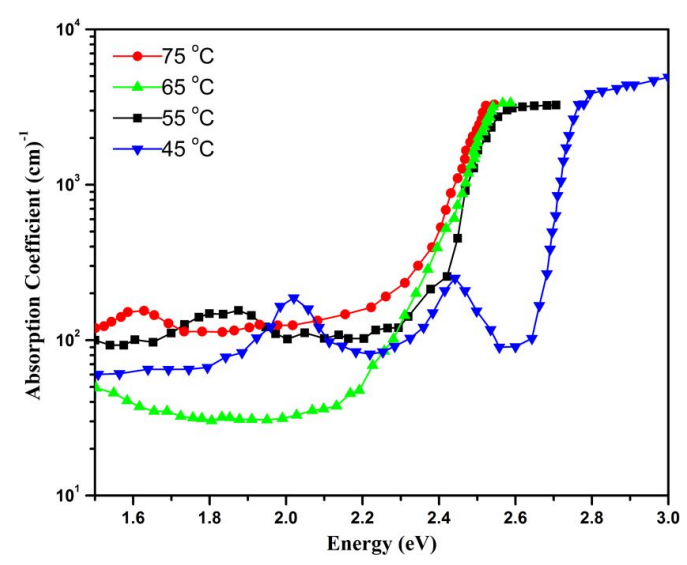

Fig. 6. Absorption coefficients of CdS nanocrystalline thin films obtained at different sols temperatures.

\subsection{I-V characteristics}

Dark and illuminated I-V characteristics of solgel grown $\mathrm{CdS}$ nanocrystalline thin films are shown in Fig. 7. It can be seen that the $\mathrm{CdS}$ nanocrystalline thin films show ohmic behavior. Electric current measurement under illumination conditions reveals a pronounced increase in conductivity of the films. Moreover, excellent photoconduction has been detected in sol-gel grown $\mathrm{CdS}$ nanocrystalline thin films. This behavior is consistent with an increase in the number of free carriers in the semiconductor under illumination, as expected from the optical properties of both bulk and nanoscale CdS [23, 24]. This confirms that the sol-gel grown $\mathrm{CdS}$ thin films show light sensitive properties. The conductivity of the films increases also with an increase in solution temperature. It can be noticed that I-V characteristics for dark and illuminated conditions exhibit 
linear behavior, which is similar to that of $\mathrm{CdS}$ thin films grown by sol-gel method [25].

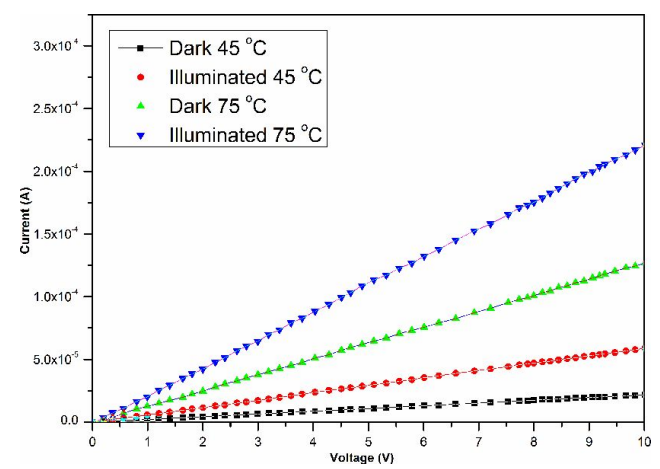

Fig. 7. I-V characteristics of CdS thin films deposited at different solution temperatures of $45^{\circ} \mathrm{C}$ and $75^{\circ} \mathrm{C}$ in dark and illuminated conditions.

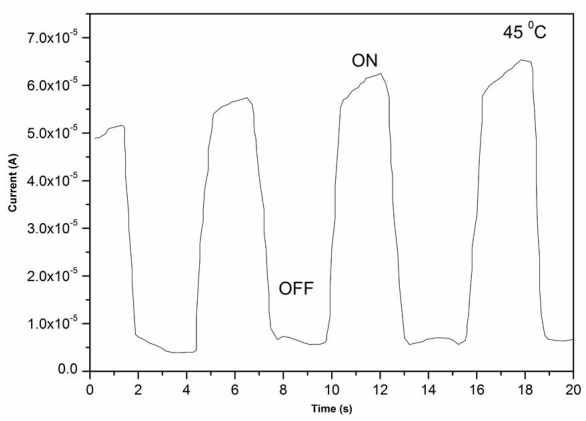

(a)

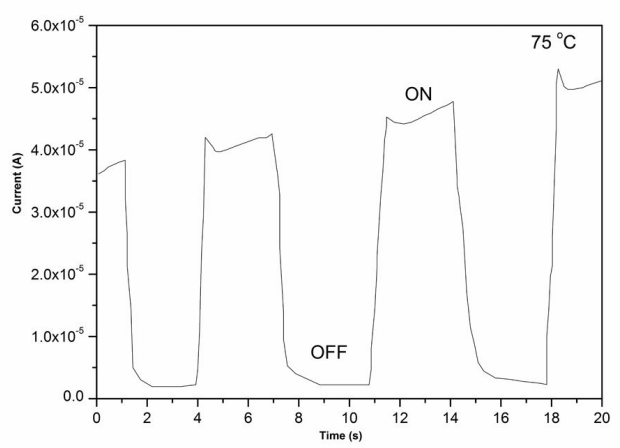

(b)

Fig. 8. Photoresponse of CdS thin films deposited at solution temperature (a) $45^{\circ} \mathrm{C}$ and (b) $75^{\circ} \mathrm{C}$.

\subsection{Photoresponse}

Photoresponse of the nanocrystalline films grown at $45{ }^{\circ} \mathrm{C}$ and $75{ }^{\circ} \mathrm{C}$ occured between low and high conductance states when the white light was turn on and off, as shown in Fig. 8. It can be seen that the response time of sol-gel grown films is fast, with a typical rise time of $1 \mathrm{~s}$ and decay time of $2.85 \mathrm{~s}$. It is quite certain that the rise time is shorter than the decay time which has been confirmed by systematic measurements. The characteristics of sol-gel grown $\mathrm{CdS}$ nanocrystalline thin films suggest that they are good candidates for optoelectronic switches, with the dark insulating states as "OFF" and the light-exposed conducting state as "ON". The mechanism of photoconductivity has already been studied previously for polycrystalline thin films, but a typical process of electronhole pair generation, trapping and recombination within CdS is complex [26]. Despite the detailed mechanism of CdS nanocrystalline thin films photoconduction has not been fully clarified, we suggest that a similar mechanism as for other polycrystalline films would be applicable. Adsorption of oxygen molecules on the surface of thin films is possible due to general properties of metal chalcogenide material. Oxygen molecules are adsorbed on the nanocrystalline thin films as negatively charged ions by capturing free electrons from ntype CdS thereby creating a depletion layer with low conductivity near the surface of the films. Since the thickness of the films is $\sim 150 \mathrm{~nm}$, only a weak depletion layer effect is observed. In the illuminated state, the absorption of light with energy greater than the band gap generates electrons in conduction band, which increases free carriers density and, in consequence, conductivity of nanocrystalline thin films. These photo-induced conductivity changes allow us to reversibly switch the nanocrystalline films between OFF and $\mathrm{ON}$ states as shown in Fig. 8, which is an optical gating phenomenon analogous to the commonly used electrical gating [27].

\section{Conclusions}

High quality $\mathrm{CdS}$ nanocrystalline thin films were fabricated successfully by sol-gel spin coating method at different solution temperatures on glass substrates. XRD analysis showed that the crystallinity of thin films improves with the increase in 
solution temperature. Crystallites size of thin films was found to be of few nanometres. Micrographs of thin films revealed compact spherical nanoparticles. The particle size assessed from the FE-SEM images was found to be $2 \mathrm{~nm}$ to $5 \mathrm{~nm}$. The particle size of the films increased with the increase in solution temperature. Band gap of thin films decreased with the increase in solution temperature. It may be due to the accelerated chemical reaction with the increase in solution temperature. The value of band gap was estimated in the range of $2.50 \mathrm{eV}$ to $2.75 \mathrm{eV}$. The absorption coefficients of thin films were calculated from UV-Vis and PDS spectroscopy. Significant amount of absorption was observed in forbidden energy gap region. I-V characteristics of the $\mathrm{CdS}$ thin films were determined under $200 \mathrm{~W}$ visible lamp.

\section{Acknowledgements}

The Author, Z.R. Khan, would like to express his gratitude to the Research Deanship, University of Ha'il, Saudi Arabia, for providing financial support.

\section{References}

[1] Husham M., Hassan Z., Selman A.M., Allam N.K., Sensor. Actuat. A-Phys., 230 (2015), 9.

[2] Challa K.K., Magnone E., Kim E.T., Mater. Lett., 85 (2012), 135.

[3] Shaikh S.U., Desale D.J., Siddiqui F.Y., Ghosh A., Birahadar R.B., GhUle A.V., Sharma R., Mater. Res. Bull., 47 (2012), 3440.

[4] Panda R., Rathore V., Rathore M., Shelke V., BADERA N., ChandRA L.S.S., JAIN D., GANGrade M., Shripati T., Ganesan V., Appl. Surf. Sci., 258 (2012), 5086.

[5] Mahdi M.A., Hassan Z., NG S.S., Hassan J.J., MOHD BAKHORI S.K., Thin Solid Films, 520 (2012), 3477.

[6] Gao T., Li Q.H., Wang T.H., Appl. Phys. Lett., 86 (2005), 173105.

[7] IKHMAYIES S.J., JUWHARI H.K., AHMAD-BITR R.N., J. Lumin., 141 (2013), 27.

[8] Ranjitha A., Muthukumarasamy N., ThamBidurai M., Velluthapillai D., BalasNDRAPRABHA R., Agilan S., J. Mater. Sci. Mater. El., 24 (2013), 3014.
[9] Salas-Villasenor A.L., Mejia I, SoteloLerma M., GuO Z.B., AlshareeF H.N., Quevedo-Lopez M.A., Semicond. Sci. Technol., 29 (2014), 85001.

[10] Hur S.G., Kim E.T., LeE J.H., Kim G.H., YoON S.G., J. Vac. Sci. Technol., B 26 (2008), 1334.

[11] Kumar S.G., RAO K.S.R K., Energ. Environ. Sci., 7 (2014), 45.

[12] XU R., WAng X., Song L., LiU W., Ji A., YAng F., Li J., Opt. Express, 20 (2012), 5061.

[13] Toyama T., Seo Y., Konishi T., OKamoto H., MoRimoto R., NishiKawa Y., Tsutsumi Y., Thin Solid Films, 555 (2014), 148.

[14] Ilahi S., Saidi F., Hamila R., Yacoubi N., AUvray L., Maaref H., Curr. Appl. Phys., 13 (2013), 610.

[15] Munirah, Khan M.S., Aziz A., Rahman S.A., Khan Z.R., Mater. Sci. Semicond. Proc., 16 (2013), 1894.

[16] Ravichandran K., Philominathan P., Appl. Surf. Sci., 255 (2009), 5736.

[17] KHAN S.A., AL-HAZMI F.S., AL-SANOSI A.M., FAidAH A.S., Yaghmour S.J., AL GHamdi A.A., Physica B, 404 (2009), 1415.

[18] Khan Z.R., Zulfequar M., Khan M.S., Mater. Sci. Eng. B-Adv., 174 (2010), 145.

[19] Ziabari A.A., Ghodsi F.E., J. Lumin., 141 (2013) 121.

[20] Hankare P.P., Chate P.A., Sathe D.J., Solid State Sci., 11 (2009) 1226.

[21] AzIz A., NArsimhan K.L., Synth. Met., 114 (2000) 133.

[22] AzIZ A., NArsimhan K.L., Synth. Met., 131 (2002) 71.

[23] Wu C., Bube R.H., J. Appl. Phys., 45 (1974), 648.

[24] Gao T., Li Q.H., Wang T.H., Appl. Phys. Lett., 86 (2005), 173105.

[25] Demira R., Gode F., Chalcogenide Lett., 12 (2015), 43.

[26] Orton J.W., Goldsmith B.J., Chapman J.A., Powell M.J., J. Appl. Phys., 53 (1982), 1602.

[27] Kind H., Yan H., Messer B., LAw M., Yang P., Adv. Mater., 14 (2002), 158.
Received 2017-02-26 Accepted 2018-02-19 